\title{
Optimization of off-null ellipsometry for air/solid interfaces
}

\author{
Yanyan Chen, ${ }^{1,2}$ Yonghong Meng, ${ }^{1}$ and Gang $\mathrm{Jin}^{1, *}$ \\ ${ }^{1}$ Institute of Mechanics, Chinese Academy of Sciences, 15 Beisihuan West Road, Beijing 100080, China \\ 2Suzhou Institute of Nano-tech and Nano-bionics, Chinese Academy of Sciences, \\ Higher Education Town Suzhou Industry Park, Suzhou 215123, China \\ ${ }^{*}$ Corresponding author: gajin@imech.ac.cn
}

Received 31 May 2007; revised 28 September 2007; accepted 23 October 2007; posted 29 October 2007 (Doc. ID 83515); published 3 December 2007

\begin{abstract}
The optimization of off-null ellipsometry is described with emphasis on the improvement of sample thickness sensitivity. Optimal conditions are dependent on azimuth angle settings of the polarizer, compensator, and analyzer in a polarizer-compensator-sample-analyzer ellipsometer arrangement. Numerical simulation utilized offers an approach to present the dependence of the sensitivity on the azimuth angle settings, from which optimal settings corresponding to the best sensitivity are derived. For a series of samples of $\mathrm{SiO}_{2}$ layer (thickness in the range of $1.8-6.5 \mathrm{~nm}$ ) on silicon substrate, the theory analysis proves that sensitivity at the optimal settings is increased 20 times compared to that at null settings used in most works, and the relationship between intensity and thickness is simplified as a linear type instead of the original nonlinear type, with the relative error reduced to $\sim 1 / 100$ at the optimal settings. Furthermore the discussion has been extended toward other factors affecting the sensitivity of the practical system, such as the linear dynamic range of the detector, the signal-to-noise ratio and the intensity from the light source, etc. Experimental results from the investigation of $\mathrm{SiO}_{2}$ layer on silicon substrate are chosen to verify the optimization. (C) 2007 Optical Society of America
\end{abstract}

OCIS codes: $120.2130,120.5240,260.2130$.

\section{Introduction}

Ellipsometry is a widely used technique for the analysis of surfaces and thin films. Many ellipsometries [1] are employed to determine the optical properties and microstructure of thin film and bulk samples or to monitor the phenomena on surfaces that involve either the growth of thin films starting from a submonolayer or the removal of such films. Among the various ellipsometry types, off-null ellipsometry is commonly used for the monitoring of phenomena on surfaces due to its fast measuring speed. This technique is similar to the reflection ellipsometry, but the main difference is that the change not only in amplitude, but also in the phase between the components parallel and perpendicular to the plane of incidence contributes to the detected signal in the off-null el-

0003-6935/07/358475-07\$15.00/0

(C) 2007 Optical Society of America lipsometry. However, only the change in amplitude is shown for the reflection ellipsometry.

Off-null ellipsometry was introduced by Trurnit in 1951 [2]. Several arrangements of off-null ellipsometer have been described by others [3-5], and in particular a polarizer-compensator-sample-analyzer (PCSA) arrangement, as shown in Fig. 1. The general principle of this arrangement is to put the polarizer, compensator, and analyzer at fixed angular positions and then to record the intensity from the detector, in which the information about the sample under measurement is contained. The detected signal records the reflection intensity from the sample surface without requiring the components and devices of the ellipsometer to be adjusted in a mechanical way as employed in other ellipsometrical techniques. This allows the measuring speed to be suitable for realtime kinetic measurements. With such a PCSA offnull ellipsometer, an in situ measurement of protein molecules adsorbed on silicon substrates was investigated by Arwin et al. [5]. During adsorption, azi- 
muth angles of the polarizer, compensator, and analyzer were fixed at special settings that reduced the detected intensity on the surface of silicon substrate to zero. Such special settings are usually called the null settings, on which a linear relationship between the thickness of protein layer (denoted by $d$ ) and the square root of the intensity (denoted by $I$ ) was given, i.e., $d=k \sqrt{I}$, where $k$ is a constant. This proportionality showed a deviation of less than $\pm 2 \%$ for $d<5 \mathrm{~nm}$.

The majority of applications of off-null ellipsometry are used to determine or follow changes of the sample properties, such as thickness and refractive index, by the corresponding change on the detected intensity. A particular challenge occurs, however, when characterization is needed for layers of material whose thickness are on the order of, or less than, the optical wavelength. In the case of layers with only a small variation in their properties, light will pass through such layers with little change in the detected intensity. To make examination of such layers possible the optical signal must be enhanced, that is to say, large intensity changes versus small changes in properties of layers to improve system sensitivity are always preferable.

A straightforward way to improve the measuring sensitivity of layers is to adjust the settings of the ellipsometer that have an effect on the detected intensity. In general, components of the ellipsometer correlating to the detected intensity can be classified mainly in the following categories: (1) Optical components, including the polarizer, the compensator, and the analyzer. The change in the azimuth angle settings of these optical components has a direct effect on the detected intensity. (2) Light source, which provides the intensity incident on the polarizer. Its choice will depend on the particular wavelength range of the ellipsometric measurements. (3) Detector. If the detector response is a linear function of the total light flux that impinges upon it, its output will be proportional to the input, which is important to the accurate quantitative analysis. The linear response range can be called the linear dynamic range of the detector. Naturally, the detected intensity is limited by this range. As with the light source, there is a wide range of detectors to choose from, dependent on the wavelength range of interest. It is necessary to consider all of these concurrently to set up the off-null ellipsometer so that the best sensitivity is obtained. However, in previous publications [5-8], the optical components are all fixed at or near the null settings, and only the optimization of azimuth angle settings has been done near the null settings, with other parts of the off-null PCSA ellipsometer fixed [6,7].

The purpose of this paper is to demonstrate the optimization from the point of view of overall setup of the off-null ellipsometer. We are particularly interested in the thickness sensitivity which is defined as

$$
r=\delta I / \delta d,
$$

where $r$ is the thickness sensitivity and $\delta I$ denotes the intensity change corresponding to the thickness change $\delta d$. This work will focus on the optimization of the off-null ellipsometer for the best sensitivity so that accurate information can be obtained. Specifically, it highlights the sensitivity depending on the azimuth angle settings of the polarizer, the compensator, and the analyzer, and discusses limitations imposed by the intensity from the light source, the dynamic range of the detector, and the signal-to-noise ratio. Theoretical discussion is represented with an emphasis on the determination of the best thickness sensitivity of silicon dioxide $\left(\mathrm{SiO}_{2}\right)$ layer on silicon substrate exposed in open air. Experimental verifications have also been made by the imaging ellipsometer whose sampling mode is the same as that of the off-null ellipsometer.

\section{Basic Theory of Off-Null Ellipsometry}

Analysis of a PCSA ellipsometer has been reported in detail by a number of authors. Herein, we briefly review the relevant theory as an introduction to the analysis that follows. Let us consider the PCSA ellipsometric system shown in Fig. 1, where the azimuth angles of the polarizer, the compensator, and the analyzer are denoted by $P, C$, and $A$, respectively. The detected intensity can be calculated from the known instrument settings and the sample properties, which may best be understood by applying a Jones vector and matrix approach using the notation convention of Azzam and Bashara, and the general expression for the detected intensity is [1],

$$
I=K L L^{*},
$$

where $*$ denotes a complex conjugation, $K=$ $\left|K_{P}\right|^{2}\left|K_{C}\right|^{2}\left|K_{A}\right|^{2} K_{D}$, where $K_{P}$ and $K_{A}$ contain information on the intensity and absolute phase of the wave emergent from the polarizer and the analyzer, respectively, $K_{C}$ accounts for the equal attenuation and phase shift along the fast and the slow axes of the

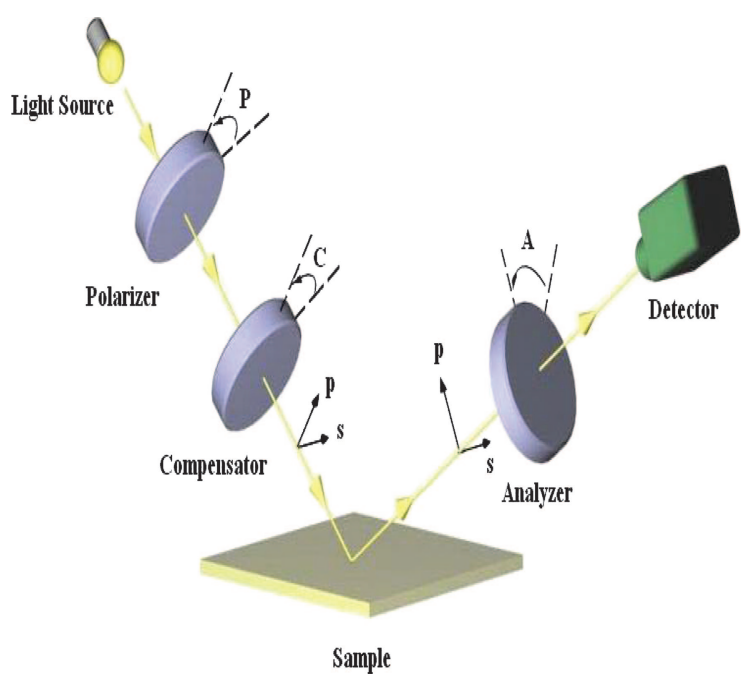

Fig. 1. (Color online) Schematic of an off-null PCSA ellipsometer. 
compensator, and $K_{D}$ is a real factor that depends on the intensity of the light beam and the nature of the detector. Throughout our discussion, we have assumed that the properties of the optical devices are uniform over the cross section of the beam, then $K$ is a constant. Another parameter $L$ in Eq. (2) is written in the form:

$$
\begin{aligned}
L= & R_{p} \cos A\left[\cos C \cos (P-C)-\rho_{c} \sin C \sin (P-C)\right] \\
& +R_{s} \sin A\left[\sin C \cos (P-C)+\rho_{c} \cos C \sin (P-C)\right] .
\end{aligned}
$$

Here $\rho_{c}$ is the slow-to-fast relative complex-amplitude transmittance of the compensator. Assuming the compensator is ideal with $\rho_{c}=-i=-\sqrt{-1}$, Eqs. (2) and (3) in combination lead to

$$
\begin{aligned}
I= & K\left(\left|R_{s}\right|^{2} / 2\right)\left\{[1+\cos 2 C \cos 2(P-C)] \cos ^{2} A \tan ^{2} \psi\right. \\
& +[1-\cos 2 C \cos 2(P-C)] \sin ^{2} A \\
& +[\sin 2 C \cos 2(P-C) \cos \Delta-\sin 2(P-C) \sin \Delta] \\
& \times \sin 2 A \tan \psi\},
\end{aligned}
$$

where the so-called ellipsometric parameters $\psi$ and $\Delta$ are defined by the ratio $\rho$ of complex-valued reflection coefficients $R_{p}$ and $R_{s}$ for light polarized parallel ( $p$ direction) and perpendicular ( $s$ direction) to the plane of incidence, respectively, namely,

$$
\rho=R_{p} / R_{s}=\tan \psi e^{i \Delta} .
$$

The amplitude ratio of $\rho$ is thus given by $\tan \psi \cdot \Delta$ is the phase difference between the reflection coefficients in the $p$ and $s$ directions. These reflection coefficients depend on the morphology and composition for the sample system with the substrate and overlayers, on layer thickness and the optical properties in the case where settings of off-null ellipsometer are fixed. In this paper we restrict ourselves to the sensitivity determined by the ellipsometer settings. For simplicity, a sample without complicated composition and morphology is chosen. $\rho$ is simplified as a function of optical constants of the ambient medium, the layers, the substrate, and thickness of the layers. For instance, in an ideal case of a layer with abrupt plane parallel-boundaries on a substrate, we have

$$
\rho=\rho\left(N_{0}, N_{1}, d, N_{2}, \phi_{0}, \lambda\right),
$$

where $N_{0}, N_{1}$, and $N_{2}$, are the refractive indices of the ambient medium, the layer, and the substrate, respectively, $\phi_{0}$ is the angle of incidence, and $\lambda$ is the wavelength. All of these have been described well by Azzam and Bashara [1], but we think it is of value to give the full picture and to provide a general understanding for the following analysis.

Deduced from Eqs. (5) and (6), functional dependence of $\psi$ and $\Delta$ on the layer thickness can be written as a complicated form [1], which makes it difficult to get the thickness sensitivity. Therefore numerical simulation is applied to simplify the functional form with other quantities known in advance, and the simplified functions are denoted $\psi(d)$ and $\Delta(d)$, respectively. We can now reformulate Eq. (4) as

$$
\begin{aligned}
I= & K\left(\left|R_{s}\right|^{2} / 2\right)\{[1+\cos 2 C \cos 2(P-C)] \\
& \times \cos ^{2} A \tan ^{2}(\psi(d)) \\
& +[1-\cos 2 C \cos 2(P-C)] \sin ^{2} A \\
& +[\sin 2 C \cos 2(P-C) \cos (\Delta(d)) \\
& -\sin 2(P-C) \sin (\Delta(d))] \sin 2 A \tan (\psi(d))\} .
\end{aligned}
$$

Equation (7) shows that the detected signal is a function of: (1) the azimuth angle $P, C$, and $A$ of the polarizer, the compensator, and the analyzer, (2) the thickness of the sample to be measured, and (3) $K$, which is related to the optical components.

By assuming a small change in $d$ and substituting $I \rightarrow I+\delta I, d \rightarrow d+\delta d$ into Eq. (7), we get the following expression:

$$
\delta I=K f\left(\left|R_{s}\right|^{2} / 2\right) \delta d,
$$

where

$$
\begin{aligned}
f= & \left\{2[1+\cos 2 C \cos 2(P-C)] \cos ^{2} A \tan (\psi(d))\right. \\
& \times\left(1+\tan ^{2}(\psi(d)) \psi^{\prime}(d)\right) \\
& -[\sin 2 C \cos 2(P-C) \sin (\Delta(d)) \\
& +\sin 2(P-C) \cos (\Delta(d))] \sin 2 A \tan (\psi(d)) \Delta^{\prime}(d) \\
& +[\sin 2 C \cos 2(P-C) \cos (\Delta(d))-\sin 2(P-C) \\
& \left.\times \sin (\Delta(d))] \sin 2 A\left(1+\tan ^{2}(\psi(d))\right) \psi^{\prime}(d)\right\} .
\end{aligned}
$$

Here $\psi^{\prime}(d)$ and $\Delta^{\prime}(d)$ represent the derivatives of $\psi(d)$ and $\Delta(d)$ to $d$, respectively.

From Eqs. (8) and (1), we may write

$$
r=\delta I / \delta d=f K\left|R_{s}\right|^{2} / 2 .
$$

In this case the sensitivity will be related to $f$ and $K$, since $R_{s}$ is determined by the properties of the sample. By recognizing that the sensitivity can be broken down into two distinct components, $f$ and $K$, a way is found to consider this problem analytically. How components of the ellipsometer affect $f$ or $K$ will be discussed in what follows.

\section{Effect of the Azimuth Angle Settings on the Sensitivity}

$K$ is a constant on the assumption that the optical components, the light source, and the dynamic linear range of the detector are all ideal and fixed, so the only varying quantity of interest is $f$. In other words, the trend of change in the sensitivity only depends on $f$, and therefore, on the settings of $P, C$, and $A$.

\section{A. Theoretical Analysis}

When the sample is changed the detailed expression of Eq. (9) also changes. Here, the sample system is the air $/ \mathrm{SiO}_{2}$ layer/silicon substrate with the layer thickness $d$ in the range of $1.8-6.5 \mathrm{~nm}$. We choose an angle 
of incidence $\phi_{0}=73.8^{\circ} . N_{0}=1.0, N_{1}=1.457$, and $N_{2}=3.858-i 0.018$ are the refractive indices of ambient, layer, and substrate, respectively, at the wavelength of $\lambda=630 \mathrm{~nm}$. Based on the sample system and these parameters, it is obvious that $\left|R_{s}\right|^{2}$ is a constant verified by the standard Fresnel formalism [1]. If all the constants are neglected, Eq. (10) can be rewritten as

$$
r \propto f .
$$

As $r$ is directly proportional to $f$, then the best positions of the azimuth angles of the optical components $(P, C$, and $A$ ) corresponding to the best sensitivity can be found theoretically by Eq. (9). We define this process as optimization, and these best positions are called optimal settings.

Equation (9) shows that $f$ is a periodic function of $P$, $C$, and $A$ with period equal to $\pi$. Considering the sign of $f$ is normally not important, and only an indication of the variation direction in the intensity to the change in thickness, we discuss the absolute value of $f$, denoted by $|f|$, and limit $P, C$, and $A$ to $[0, \pi / 2]$ in this paper. According to the ellipsometry theory in Azzam and Bashara's book, the John vector is represented as

$$
E_{c o}{ }^{f s}=K_{c} K_{p}\left[\begin{array}{c}
\cos (P-C) \\
\rho_{c} \sin (P-C)
\end{array}\right]
$$

for the output light from the compensator, which means $E_{c o}{ }^{f s}$ is relative to the difference between $P$ and $C(P-C)$. Any value of $P-C$ can be obtained by varying $P$ at a fixed $C$. For simplification, $C$ is fixed at $45^{\circ}$, and only $P$ and $A$ are discussed in this paper. Thus Eq. (9) can be simplified as

$$
\begin{aligned}
f= & {\left[2 \cos ^{2} A \tan (\psi(d))+\sin (2 P+\Delta(d)) \sin 2 A\right] } \\
& \times\left[1+\tan ^{2}(\psi(d))\right] \psi^{\prime}(d) \\
& +\cos (2 P+\Delta(d)) \sin 2 A \tan (\psi(d)) \Delta^{\prime}(d),
\end{aligned}
$$

from which it is readily seen how $|f|$ depends on $P$ and $A$.

As an example, Fig. 2 shows a result from a simulation of Eq. (12) for the $\mathrm{SiO}_{2}$ layer on silicon substrate. The parameters are determined as follows: in terms of above instrument setting parameters, the sample system, and by the standard Fresnel formalism [1], it is found that $\Delta$ is proportional to the $\mathrm{SiO}_{2}$ layer thickness $d$, and a square relationship exists between $\psi$ and $d$, with the deviation less than $\pm 1 \%$ for $d<6.5 \mathrm{~nm}$. These relationships can be expressed as

$$
\begin{aligned}
& \psi(d)=a_{\psi} d^{2}+k_{\psi} d+c_{\psi}, \\
& \Delta(d)=a_{\Delta} d^{2}+k_{\Delta} d+c_{\Delta},
\end{aligned}
$$

where $a_{\psi}=4 \times 10^{-4}, a_{\Delta}=6.85 \times 10^{-3}, k_{\psi}=1.6$ $\times 10^{-3}, k_{\Delta}=-1.697 \times 10^{-1}, c_{\psi}=5.97 \times 10^{-2}$, and

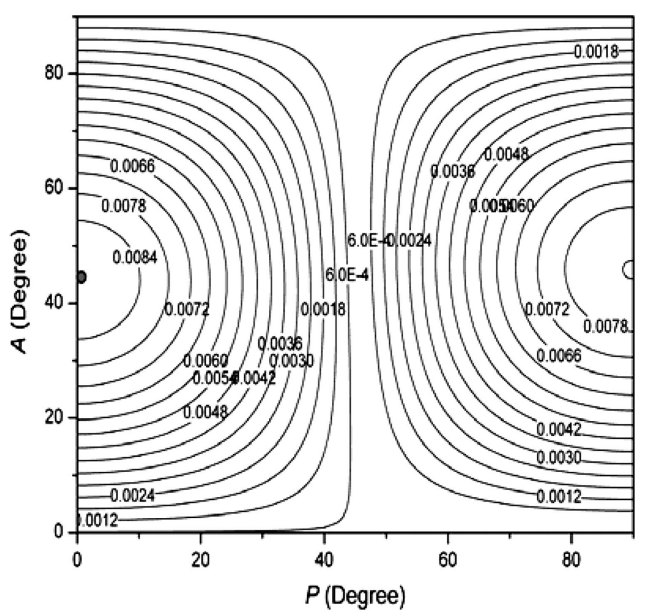

Fig. 2. Simulated contour map of the absolute value of $f$ for the $\mathrm{SiO}_{2}$ layer on silicon substrate sample with $d=3 \mathrm{~nm}$, related to $P$ and $A$ varied from $0^{\circ}$ to $90^{\circ}$ with step $1^{\circ}$, respectively. Here the values marked in it represent $|f|$. The fact $r \propto f$ makes it obvious that the proper azimuth settings can be chosen for different sensitivity, and the optimal settings corresponding to the highest sensitivity are the azimuth angle settings which correspond to $|f|_{\max }$, shown as the gray dot, i.e., at $P=0.19^{\circ}$ and $A=44.67^{\circ}$ in this graph.

$c_{\Delta}=3.1107$. The unit corresponding to $a_{\psi}$ and $a_{\Delta}$ is $\mathrm{rad} / \mathrm{nm}^{2}$, to $k_{\psi}$ and $k_{\Delta}$ is $\mathrm{rad} / \mathrm{nm}$, to $c_{\psi}$ and $c_{\Delta}$ is $\mathrm{nm}$. Substitution of Eq. (13) into Eq. (12) and for a $\mathrm{SiO}_{2}$ layer with $d=3 \mathrm{~nm}$, the dependence of $|f|$ on $P$ and $A$ is given, as shown by the contour map in Fig. 2, where the values marked in it represent $|f|$. Equation (11) makes it obvious that the optimal settings corresponding to the highest sensitivity are the azimuth angle settings, which correspond to the maximum of $|f|$ (denoted by $|f|_{\max }$ ), as shown by the gray dot, i.e., at $P=0.19^{\circ}$ and $A=44.67^{\circ}$ in this graph.

Note that $f$ is a function of $P$ and $A$ shown in Eq. (12), we arrive at an expression for the $P$ and $A$ corresponding to maximum sensitivity by the extreme method:

$$
\begin{array}{r}
\frac{\partial f}{\partial P}=\frac{\partial f}{\partial A}=0, \\
\frac{\partial^{2} f}{\partial P^{2}} \frac{\partial^{2} f}{\partial A^{2}}-\left(\frac{\partial^{2} f}{\partial P \partial A}\right)^{2}>0,
\end{array}
$$

giving

$$
\begin{aligned}
& P_{n}=\frac{1}{2}\left[n \pi+\arctan \left(m_{1} / m_{2}\right)-\Delta(d)\right] \\
& A_{n}=\frac{1}{2}\left[n \pi \pm \operatorname{arccot}\left(\frac{m_{1} \tan \psi(d)}{\sqrt{m_{1}^{2}+m_{2}^{2}}}\right)\right],
\end{aligned}
$$

where the azimuth angles of the polarizer and the analyzer corresponding to the extreme are denoted by $P_{n}$ and $A_{n}(n=1,2, \ldots, n \in z, z$ denotes the integer), $m_{1}=\left[1+\tan ^{2}(\psi(d))\right] \psi^{\prime}(d), m_{2}=\tan (\psi(d)) \Delta^{\prime}(d)$. 
Since $P_{n}$ and $A_{n}$ depend on $d$, this means if a series of samples with different thicknesses are measured with the off-null ellipsometer, measurements must be carried out one by one by mechanical adjustment to the optimal settings for each sample. However, the off-null ellipsometer is used mainly to determine or follow changes of the sample properties in the kinetic process, therefore it is not normally a practical solution to readjust the settings every time. One feasible method is to set the azimuth angles at the middle settings of a series corresponding to a certain thickness range of sample. The middle settings may be variable as the thickness range of the sample is different.

Another aspect we should consider after the azimuth angles have been determined is the functional relationship between the intensity $I$ and the thickness $d$, which is usually applied for quantitative measurement. For instance, off-null ellipsometry can be used to follow fast kinetics during a film growth. If the relationship expression between $I$ and $d$ is simple, such as linear, the proportionality constant in the relationship is obtained with the final thickness measured at a steady state. The actual kinetics of film growth, that is to say, the thickness of film growth, can then be calculated from the recorded intensity by this known relationship. However, for an expression like Eq. (7), it is not convenient for quantitative measurements since multicoefficients exist in the expression, making it complicated to determine the expression. The expression therefore, should be simplified further by fitting the expressional curve.

Examples are given at three azimuth angle settings to show different sensitivities and their simplified relationships between $I$ and $d$. Now that the azimuth angles in most works are fixed at or close to the null settings, it is necessary to consider the null settings in this work. Here, the null settings chosen correspond to the azimuth angles that fulfill the extinction of light leaving the analyzer on the $\mathrm{SiO}_{2}$ layer with $d=1.8 \mathrm{~nm}$. The optimal settings are the settings mentioned above, and better settings are chosen at any other positions as a comparison.

Based on the standard Fresnel formalism and the exact equations of ellipsometry Eq. (4), we show in Fig. 3 the computed curves for $I$ versus $d$ in one thickness period of $\mathrm{SiO}_{2}$ layer at above three settings. It must be mentioned that all of the curves are obtained on the assumption of $K=1$. The change of slopes corresponding to the same thickness range in three curves, or to the different thickness ranges in one curve, indicates clearly the difference in sensitivity, which reminds us to choose the appropriate settings according to different samples in practical applications. For the part of $d<6.5 \mathrm{~nm}$, the expression of the relationship between $I$ and $d$ can be determined by simulating these curves within acceptable relative error levels. Detailed results are shown in Table 1 , including settings of $P$ and $A$, the sensitivities, as well as the type of relationship. Note that curve (a) with respect to the null settings is also simplified as a linear relationship with relative error $>10 \%$, and is not accepted, so a square relationship is

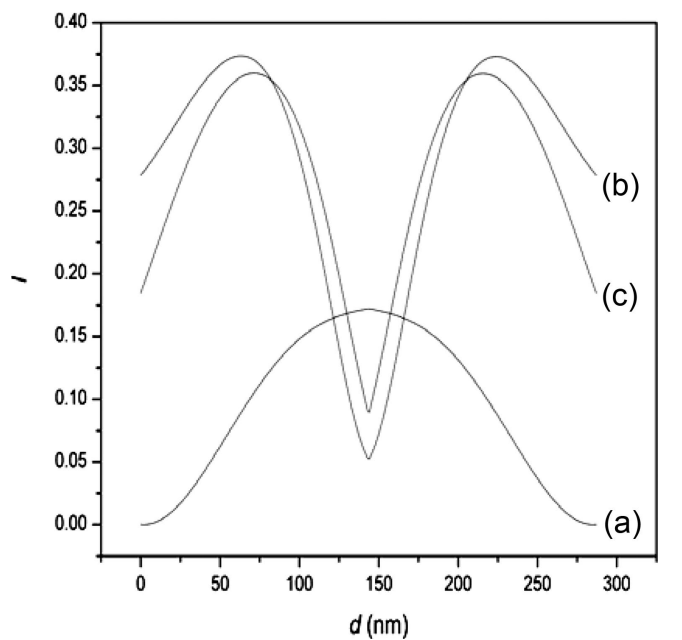

Fig. 3. Intensity as a function of the $\mathrm{SiO}_{2}$ thickness in one thickness period for the air- $\mathrm{SiO}_{2}-\mathrm{Si}$ system at (a) null settings at where $A=3.83^{\circ}, P=54.10^{\circ}$, (b) better settings at where $A=62.82^{\circ}$, $P=28.86^{\circ}$, and (c) optimal settings at where $A=44.67^{\circ}$, $P=0.19^{\circ}$. It is based on the assumption of $K=1$ and obtained by Eq. (2). For the part of $d<6.5 \mathrm{~nm}$, the difference among the three curves is obvious, and the fitted expression is $I_{a}=3.4 \times 10^{-5} d^{2}$ $-1.29 \times 10^{-4} d+1.25 \times 10^{-4}, I_{b}=1.706 \times 10^{-3} d+0.278$, and $I_{c}=3.63 \times 10^{-3} d+0.185$, respectively. The detailed explanations are shown in Table 1 .

then considered and square expression $I_{a}=3.4 \times$ $10^{-5} d^{2}-1.29 \times 10^{-4} d+1.25 \times 10^{-4}$ with relative error $\sim 1 \%$ is obtained. Curve (b) with respect to better settings and (c) with respect to the optimal settings are all linear curves with $I_{b}=1.706 \times 10^{-3} \mathrm{~d}$ +0.278 and $I_{c}=3.63 \times 10^{-3} d+0.185$, respectively. Clearly, it can be seen that the lowest sensitivity, $r_{a}$, is obtained near the null settings, as well as the nonlinear relationship with the largest error. On the contrary, curve (c) at optimal settings corresponds to a simple linear relationship with the least error and the highest sensitivity, $r_{c}$. It is also found the sensitivity is increased more than 20 times and the relative error is reduced to $\sim 1 / 100$ for the optimal settings compared with the null settings. In comparing curve (b) and (c), both are linear curves with the same error level, but $r_{b}$ is decreased by $50 \%$ compared with $r_{c}$. We also investigate the relationship at other

Table 1. Azimuth Angle Settings, Relationship, Relative Error, and Sensitivity of Three Different Settings are Shown Corresponding to Fig. $3^{a}$

\begin{tabular}{|c|c|c|c|c|c|}
\hline \multirow[b]{2}{*}{ Type } & \multicolumn{2}{|c|}{$\begin{array}{l}\text { Azimuth } \\
\text { Angle } \\
\text { Setting } \\
\left(^{\circ}\right)\end{array}$} & \multirow[b]{2}{*}{ Relationship } & \multirow{2}{*}{$\begin{array}{c}\text { Relative Error } \\
(\%)\end{array}$} & \multirow[b]{2}{*}{$r\left(\times 10^{-3}\right)$} \\
\hline & $P$ & $A$ & & & \\
\hline \multirow[t]{2}{*}{ a } & 54.10 & 3.83 & Linear & $>10$ & 0.149 \\
\hline & & & Square & 1 & \\
\hline $\mathrm{b}$ & 28.86 & 62.82 & Linear & 0.02 & 1.706 \\
\hline $\mathrm{c}$ & 0.19 & 44.67 & Linear & 0.02 & 3.63 \\
\hline
\end{tabular}

${ }^{a}$ The unit of $r$ is lux $/ \mathrm{nm}$. 
settings, and find that the linear relationship is obtained with higher accuracy near the optimal settings and with lower accuracy near the null settings.

\section{B. Experimental Verification}

For verifying the above analysis, some experiments on samples of $\mathrm{SiO}_{2}$ layers/Si substrate are performed with a PCSA imaging ellipsometer, which has the same sampling technique of the off-null ellipsometer. It is an instrument with an expanded parallel light beam instead of a conventional narrow beam. The light intensity reflected from the sample under measurement will then be detected by a charge-coupleddevice (CCD) camera. The CCD video camera replaces the traditional photomultiplier or photodiode detector for imaging purpose. In this way, every point in the field of view can be measured at the same time, which provides the way for multiple layer measurements simultaneously. The detailed technique has been described in the literature [8].

In our experimental setup, a $75 \mathrm{~W}$ xenon arc lamp and a collimating system are used to provide an expanded parallel probe beam, whose intensity fits to the linear dynamic range of the detector. The polarizer and the analyzer are dichroic sheet polarizers with the extinction ratio $10^{-4}$, and a mica retardation of a quarter-wave plate is used as the compensator. The detected-results are stored as the images in grayscale format (8 bit, 256 gray scales) for further evaluation by an image processing program. The CCD camera is a Sony XC-ST30 CCD B/W video camera, and it has been proved the dynamic region of the detector is linear when the gray value is among 15230 in the imaging ellipsometer.

Samples are etched in hydrofluoric acid to create the $\mathrm{SiO}_{2}$ layer thicknesses in the range of $1.8-6.5 \mathrm{~nm}$. The original $\mathrm{SiO}_{2}$ layer with $d=64 \mathrm{~nm}$ on silicon wafer is dipped into the hydrofluoric acid (diluted to $1 / 500$ ) and etched within 15-30 min to get a series of $\mathrm{SiO}_{2}$ layers with thickness in the range of 1.8-6.5 nm. Each thickness of $\mathrm{SiO}_{2}$ layer is calibrated with an ellipsometer of rotating analyzer type (SE 400, SENTECH, Germany) equipped with a He-Ne laser (wavelength $632.8 \mathrm{~nm}$ ). The measurements are carried out at an angle of incidence of $70^{\circ}$. With the fixed refractive index of $\mathrm{SiO}_{2}$ layer $(n=1.457)$ on silicon substrate known, each sample is measured three times and the measurement error is $\sim 0.1 \mathrm{~nm}$. These samples are measured with the imaging ellipsometer at the same azimuth angle settings mentioned above, and experimental results are shown in Fig. 4. Each result is an average of 100 measurements, and the error on measured intensity is decreased to less than $0.5 \%$. The difference in gray scales between 6.5 and $1.8 \mathrm{~nm} \mathrm{SiO}_{2}$ layers is below 0.5 at the null settings, which is so difficult to recognize the difference in layers that the experimental results do not show in Fig. 4. Curves (b) and (c) are obtained at the other two settings with fitted expression $I_{b}=1.158 d+216.51$ and $I_{c}=2.344 d$ +135.99 , respectively. Their relative errors are all



Fig. 4. Gray scales with respect to the thickness of $\mathrm{SiO}_{2}$ layer in experiment. The dotted line is the experimental results and the solid line is the approximate curve with the corresponding expression (b) $I_{b}=1.158 d+216.51$, in which the azimuth setting is the same as in Fig. 3(b), and (c) $I_{c}=2.344 d+135.99$, in which the azimuth settings are the same as in Fig. 3(c).

within $\pm 0.2 \%$. Note that the sensitivities are different from those in Table 1, the reason is $K$ in Eq. (1) is not discussed in simulation analysis, but included in experimental results. However, $r_{b}=1.16 / K$ is about a half $r_{c}=2.34 / K$, which also proves the consistency of theory and experiment.

\section{Discussion on the Sensitivity}

Based on $K=\left|K_{P}\right|^{2}\left|K_{C}\right|^{2}\left|K_{A}\right|^{2} K_{D}$, the sensitivity can be improved further by setting the light source to provide higher intensity and using a detector with a wider linear dynamic range. However, the optimal sensitivity in theory may not be applied in an experimental system where the light source and the detector are all fixed. It can be demonstrated by the simulation in Fig. 3. If the linear dynamic range is wide enough to detect the maximum intensity in Fig. 3 , all the layers in the whole thickness range can be detected, and the best sensitivity is still obtained at setting (c) for $\mathrm{SiO}_{2}$ layers in the range of $1.8-6.5 \mathrm{~nm}$. Otherwise, if the intensity corresponding to curve (c) is out of the linear dynamic range, the detected intensity of $\mathrm{SiO}_{2}$ layer must be lowered so that it fits the range. There are two ways to achieve this. One is to keep setting (c) but decrease the intensity incident on the polarizer by adding a component of intensity attenuation, for example, a ground glass. Another is to keep the intensity constant but readjust the azimuth angle settings of the polarizer and analyzer. In comparing the two methods, one found that the sensitivity would be decreased evidently with the decrease of the intensity incident on the polarizer. Therefore, an alternative way is to adjust the azimuth angles of the polarizer and analyzer based on invariable intensity for the best sensitivity in a practical system.

If the best sensitivity in theory is invalid in the practical system, the best sensitivity in the practical 


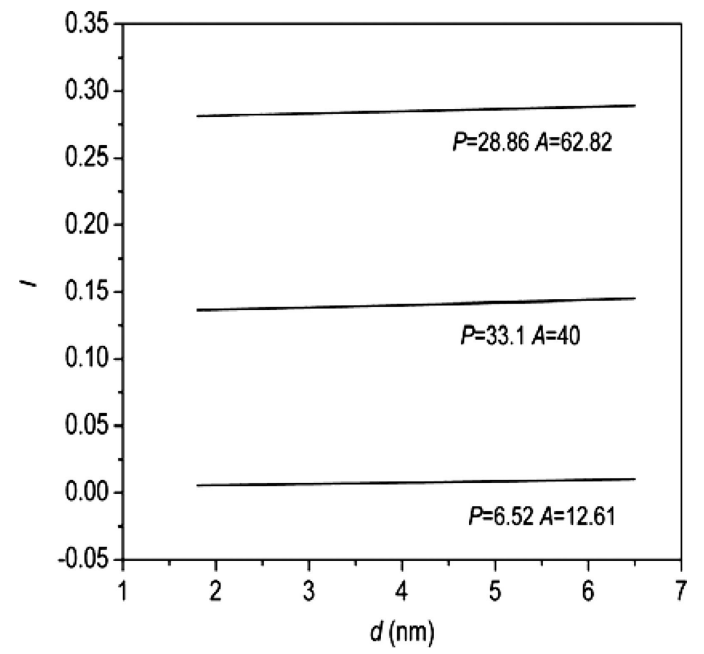

Fig. 5. Three computed curves, in which $I$ versus $d$ in the thickness range of $1.8-6.5 \mathrm{~nm}$ of $\mathrm{SiO}_{2}$ layer at three azimuth angle settings are shown. The detailed azimuth angles are noted in the graph. The parallelism in these curves proves the sensitivities are the same, but the difference in the intensity $I$ at three settings indicates that the settings corresponding to the lowest intensity should be chosen to measure samples in order to avoid the intensity out of the dynamic range.

system must correspond to one of the theoretical sensitivities between the optimal sensitivity and the lowest. From Fig. 2 it can be seen that there are multiple azimuth angle settings of the polarizer and the analyzer corresponding to such a sensitivity. To further demonstrate this, three computed curves of $I$ versus $d$, for $\mathrm{SiO}_{2}$ layers in the range of $1.8-6.5 \mathrm{~nm}$, at three settings corresponding to one sensitivity, are shown in Fig. 5. The parallel nature of these curves proves sensitivities are identical, although the intensity is different. Therefore, for the detector with a lower dynamic range, one can choose the settings corresponding to the lowest intensity in order to achieve a valid measurement.

Another quantity under consideration from the practical application point of view is the signal-to-noise ratio, defined as the ratio of detected signal and noise. In practice noise includes the depolarizing tendencies of the optical devices encountered by the light beam, any stray-light pickup, as well as the dark current of the detector. Some components may require more careful selection for the system to decrease the noise. Once they are selected, noise is regarded as a constant. This means that the higher the detected intensity, the higher the signal-to-noise ratio. Out of question, the lowest signal-to-noise ratio is obtained at the null settings, and in comparison, a higher signal-to-noise ratio is observed at the optimal settings.

All of the discussion mentioned above is concerned with the factors relating to sensitivity. Another question for us to think over is the relationship between the intensity and the thickness, since it is helpful for quantitative measurement. Combining the analysis mentioned above and the constant $K$, it can be concluded that the type of relationship is only deter- mined by azimuth angle settings. In other words, the types of relationships obtained at the three settings above are still invariable even though the light source and the dynamic range of detector are changed. Thus, the azimuth angles of the polarizer and the analyzer must be set far away from the null settings when a simple linear relationship is required, which depends on the requirement of practical applications.

\section{Conclusion}

The optimal sensitivity of an off-null ellipsometer has been presented in detail, with particular emphasis on the theoretical analysis of the azimuth angles of the polarizer and the analyzer so that the optimal sensitivity is obtained. By comparison between the optimal settings and the null settings, 1 order of magnitude increase in the sensitivity is observed. The potential applications of off-null ellipsometry can be extended further based on the optimal sensitivity. Moreover, combined theoretical analysis with some factors, like the dynamic range of the detector, the intensity provided by the light source and the ratio of the signal to noise, how to get the best sensitivity in a practical system is also discussed. The sample system of the air $/ \mathrm{SiO}_{2}$ layer/silicon substrate with the layer thickness $d$ in the range of $1.8-6.5 \mathrm{~nm}$ is studied as one application example. In fact, the methodology provided here can also be applied to the sample system with other thickness ranges, some different material (such as organic layer, oxide layer, etc.), or in liquid ambient.

The National Natural Science Foundation of China and The Chinese Academy of Sciences (KJCX2.YW.M02 and M04) are gratefully thanked for their support.

\section{References}

1. R. M. A. Azzam and N. M. Bashara, Ellipsometry and Polarized Light (North-Holland, 1977).

2. H. J. Trumit, "Monomolecular layers," in A Symposium Presented on December 27, 1951, at the Philadelphia Meeting of the Association for the Advancement of Science, H. Sobotka, ed. (University of Chicago Press, 1956), p. 321.

3. B. D. Cahan, J. Horkans, and E. Yeager, "Modulation ellipsometry and its application to the study of the electrode-electrolyte interface," Surf. Sci. 37, 559-567 (1973).

4. J. O'M. Bockris, M. Genshaw, and V. Brusic, "Mechanism of film growth and passivation of iron as indicated by transient ellipsometry," Symp. Faraday Soc. 4, 177-191 (1970).

5. H. Arwin, S. Welin-Klintström, and R. Jansson, "Off-null ellipsometry revisited: basic consideration for measuring surface concentrations at solid/liquid interfaces," J. Colloid Interface Sci. 156, 377-382 (1993)

6. G. L. Wang, H. Arwin, and R. Jansson, "Optimization of azimuth angle settings in polarizer-compensator-sample-analyzer off null ellipsometry," Appl. Opt. 42, 38-44 (2003).

7. G. L. Wang, H. Arwin, and R. Jansson, "Optimization of off null ellipsometry in sensor applications," Appl. Opt. 43, 2000-2005 (2004).

8. G. Jin, R. Jansson, and H. Arwin, "Imaging ellipsometry revisited: developments for visualization of thin transparent layers on silicon substrates," Rev. Sci. Instrum. 67, 2930-2936 (1996). 